\title{
Rapid Quantitative Detection of Salmonella enterica Using Fluorescence In Situ
}

\section{Hybridization with Filter-cultivation (FISHFC) Method}

\author{
Shigemasa Shimizu ${ }^{1}$, Ryouhei $\mathrm{AoI}^{2}$, Yui OsAnAI ${ }^{2}$, Yuji $\mathrm{KAWAI}^{2}$ and Koji YAMAZAKI ${ }^{2 *}$ \\ ${ }^{1}$ Division of Processing and Utilization, Abashiri Fisheries Research Institute, Hokkaido Research Organization, 7-8-5, Minato, Monbetsu, \\ Hokkaido 094-0011, Japan \\ ${ }^{2}$ Division of Marine Life Science, Faculty of Fisheries Sciences, Hokkaido University, 3-1-1, Minato, Hakodate, Hokkaido 041-8611, Japan
}

Received June 20, 2012; Accepted October 12, 2012

Specific detection and enumeration of Salmonella enterica in food using conventional culture-based methods (CCBM) are time consuming and labor intensive. This study was conducted to develop a rapid $S$. enterica detection and enumeration method by combining fluorescence in situ hybridization (FISH) with micro-colony formation culture (FISHFC). Specificity tests of the SAL343 probe for $S$. enterica detection revealed that SAL343-associated fluorescent micro-colonies were observed specifically for $S$. enterica, but not for any other organisms. This finding suggests that SAL343 is highly specific for detecting $S$. enterica using FISHFC. For validation, FISHFC with SAL343 was compared to CCBM, with multiple selective agar, using spiked food samples; no significant differences in enumeration were found between FISHFC and CCBM $(p>0.05)$. The FISHFC method allowed enumeration of $S$. enterica within $10 \mathrm{~h}$ while CCBM allowed enumeration within 5 days. Therefore, the FISHFC method has potential application for more rapid and specific enumeration of $S$. enterica in food samples compared to other available methods.

Keywords: Salmonella enterica, fluorescence in situ hybridization, FISHFC, rapid detection

\section{Introduction}

Salmonella are Gram-negative, facultatively anaerobic, rod-shaped bacteria that exist ubiquitously in the environment and are commonly found in water, animal intestine, reptilian skin and food. Salmonella also causes salmonellosis in humans worldwide due to the consumption of contaminated food such as fresh fruits and vegetables (Matthews, 2006). The genus Salmonella currently consists of two species: $S$. enterica and S. bongori. S. enterica is comprised of six subspecies: enterica, salamae, arizonae, diarizonae, houtenae and indica, which are further subdivided into more than 2,500 serotypes (Agbaja et al., 2011; Su and Chiu, 2007). Most of the Salmonella responsible for disease in humans and animals are members of S. enterica. S. enterica serotype Enteritidis and Typhimurium are two of the main sources of human food poisoning. Although $S$. bongori has been reported to infect humans, it is generally associated with cold-blooded animals (Giammanco et al., 2002). Therefore, S. enterica are clinically important pathogens of humans.

*To whom correspondence should be addressed. E-mail: yamasaki@fish.hokudai.ac.jp
Routine methods for enumerating Salmonella are traditional microbiological methods based on plating using selective agar and biochemical identification. However, the biochemical characteristics of some isolates may differ from the common phenotypic properties of typical strains. Moreover, it is recommended that multiple selective agars be used for Salmonella detection in food samples (Nye et al., 2002). Thus, the conventional methods for detecting Salmonella are time-consuming and labor-intensive, taking at least several days to obtain the final results (Kim et al., 2007). To overcome technical problems associated with Salmonella detection, a number of molecular-based methods, such as PCR, quantitative PCR (qPCR), enzyme-linked immunosorbent assay (ELISA) and single-cell fluorescence in situ hybridization (FISH), have been developed (Kawasaki et al., 2010; Goodridge et al., 2003; Fang et al., 2003). These cultureindependent methods can achieve faster detection; however, a serious limitation of some methods is the detection of both live and dead cells.

FISH is a reliable technique for identifying and localizing the presence of specific RNAs or DNAs in cells, and this technology has been applied to the detection of specific 
microorganisms in environmental, clinical and food samples. However, FISH has a number of practical limitations. For example, environmental and food particles interfere with bacterial single-cell detection using epi-fluorescence microscopic observation. Furthermore, the aggregation of bacterial cells impedes the accurate determination of bacterial counts. In addition, bacterial cell detection using FISH technology must typically be performed microscopically under high magnification ( $>1,000$-fold), meaning that many microscopic fields must be observed for precise bacterial detection in specimens.

To overcome these challenges, a superior method combining the FISH technique and brief filter-cultivation, known as FISHFC (fluorescence in situ hybridization with filter cultivation), was developed to enumerate viable Enterobacteriaceae (Ootsubo et al., 2003). The FISHFC method makes it possible to enumerate only viable targeted microorganisms from food and environmental samples, both rapidly and specifically. FISHFC has several advantages, including resistance to sample debris causing noise when differentiating bacterial micro-colonies with a specific probe, detectability of viable bacteria, rapidity (the overall reaction is completed within $15 \mathrm{~h}$ ), and a high detection limit (10 CFU/mL food homogenate) (Sawabe et al., 2009). Thus, FISHFC has advantages over many other rapid detection methods such as qPCR, ELISA and FISH. Previously, we demonstrated the accurate enumeration of Listeria spp., L. monocytogenes and Clostridium perfringens in food samples using FISHFC (Fuchizawa et al., 2008; Shimizu et al., 2009).

The aim of this study was to develop the probe SAL343 for FISHFC detection of $S$. enterica and to establish the FISHFC protocol for viable detection of $S$. enterica in food samples.

\section{Material and methods}

Bacterial strains A list of bacterial strains used in this study is shown in Table 1. All strains were maintained on tryptic soy agar (TSA, BD, Sparks, MD, USA at $4^{\circ} \mathrm{C}$. Fresh cultures were prepared by inoculating tryptic soy broth (TSB, $\mathrm{BD}, \mathrm{USA}$ ) at $30^{\circ} \mathrm{C}$ or $37^{\circ} \mathrm{C}$ for $24 \mathrm{~h}$ aerobically, except for halophilic bacteria, which were maintained on TSA containing $3 \% \mathrm{NaCl}$ and grown in TSB containing 3\% NaCl.

Design of oligonucleotide probe The rRNA-targeted oligonucleotide probe for specific Salmonella detection, SAL343 (5'- GCT CAC AGC ACA TGC GCT TTT G -3'), was designed using the $23 \mathrm{~S}$ rRNA region of $S$. Enteritidis (EU146952) found in the rRNA database (Genbank/ EMBL/DDBJ). The oligonucleotide probe was optimized for Gibbs's free energy of DNA-RNA hybridization (Yilmaz and Noguera, 2004). SAL343 specificity was confirmed by comparison with $16 \mathrm{~S}$ and $23 \mathrm{~S}$ rRNA sequences using probeCheck software (Loy et al., 2008). SAL343 was labeled at the 5'-end with Alexa Fluor 546 ${ }^{\circledR}$ (Ex; 556 nm, Em; 573 nm) (Japan Bio Services Co. Ltd., Saitama, Japan). A universal oligonucleotide probe, EUB338 (5'- GCT GCC TCC CGTAGG AGT -3) (Amann et al., 1990), was used as the positive hybridization control. EUB338 was labeled at the 5'-end with Alexa Fluor $488^{\circledR}$ (Ex; 496 nm, Em; 519 nm).

Probe SAL343 specificity in the FISHFC method For the specificity test of SAL343, FISHFC was performed on Gram-negative bacteria related to Salmonella species. Bacterial fresh cultures were centrifuged at $10,000 \times g$ for 2 min in a micro-centrifuge tube and washed with buffered peptone water $(\mathrm{pH} 7.0)$. An aliquot $(100 \mu \mathrm{L})$ of each diluted bacterial culture was inoculated into $4 \mathrm{~mL}$ of TSB and then pressfiltered using 37-mm monitor units (Advantec Co. Ltd., Tokyo, Japan) fitted with hydrophilic polypropylene membrane filters (pore size; $0.45 \mu \mathrm{m}$, Pall Corp., East Hills, NY, USA). After formation of micro-colonies by incubation at $30^{\circ} \mathrm{C}$ or $37^{\circ} \mathrm{C}$ for $6-9 \mathrm{~h}$, membrane filters were fixed by ethanol spraying $(99.5 \%)$ at room temperature $(\mathrm{RT})$ for $15 \mathrm{~min}$. The membrane filters were air dried and hybridized in $1 \mathrm{~mL}$ of pre-warmed $\left(46^{\circ} \mathrm{C}\right)$ hybridization buffer $(30 \%$ formamide, $0.9 \mathrm{M} \mathrm{NaCl}, 0.01 \%$ sodium dodecyl sulfate [SDS], $0.02 \mathrm{M}$ Tris- $\mathrm{HCl}$ and $10 \mu \mathrm{M} \mathrm{SAL343} \mathrm{[pH} \mathrm{7.4])} \mathrm{at} 46^{\circ} \mathrm{C}$ for $60 \mathrm{~min}$. The membrane filters were then washed with $2 \mathrm{~mL}$ of prewarmed $\left(46^{\circ} \mathrm{C}\right)$ wash buffer $(0.18 \mathrm{M} \mathrm{NaCl}, 0.01 \%$ SDS and $0.02 \mathrm{M}$ Tris- $\mathrm{HCl}\left[\mathrm{pH} \mathrm{7.4])}\right.$ at $46^{\circ} \mathrm{C}$ for $15 \mathrm{~min}$, rinsed with sterile distilled water (SDW) and air dried again. The microcolonies on the membrane filters were observed using epifluorescence microscopy (Nikon E600 [Nikon Corp., Kawasaki, Japan], objective lens; Plan fluor $\times 4$, fluorescent filter; XF204 [Omega Optical, Brattleboro, VT, USA] for Alexa Fluor $546^{\circledR}$ and XF202 [Omega] for Alexa Fluor 488 ${ }^{\circledR}$ ). Images were captured using a digital camera D50 (Nikon).

Optimization of cultivation time in FISHFC Optimal cultivation time was determined to obtain adequate colony size for epi-fluorescence microscopic observation with a 4-fold objective lens. Aliquots $(0.1 \mathrm{~mL})$ of $S$. Enteritidis NBRC3313, S. Typhimurium IID1000 and $S$. Infantis ATCC51741 cultures were inoculated into $4 \mathrm{~mL}$ of Mannitol lysine crystal violet brilliant green (MLCB) broth (peptone; $10.0 \mathrm{~g}$, yeast extract; $5.0 \mathrm{~g}$, Lab Lemco powder; $2.0 \mathrm{~g}$, sodium chloride; $4.0 \mathrm{~g}$, mannitol; $3.0 \mathrm{~g}$, L-lysine hydrochloride; $5.0 \mathrm{~g}$, sodium thiosulfate; $4.0 \mathrm{~g}$, ferric ammonium citrate; 1.0 $\mathrm{g}$, brilliant green; $12.5 \mathrm{mg}$, crystal violet; $0.01 \mathrm{~g}, \mathrm{pH} 6.8 \pm 0.2$ ) and then press-filtered using 37-mm monitor units (Advantec) fitted with hydrophilic polypropylene membrane filters (Pall). After incubation at $37^{\circ} \mathrm{C}$ for $6-9 \mathrm{~h}$, FISH was performed as described above. The micro-colonies on the membrane filters 
Table 1. Probe specificity of SAL343 for Salmonella detection.

\begin{tabular}{|c|c|c|c|}
\hline Bacteria & Strain & EUB338 & SAL343 \\
\hline \multicolumn{4}{|l|}{$<$ Salmonella $>$} \\
\hline Salmonella enterica subsp. enterica Enteritidis & NBRC3313 & + & + \\
\hline S. enterica subsp. enterica Enteritidis & IID604 & + & + \\
\hline S. enterica subsp. enterica Enteritidis & RIMD1933001 & + & + \\
\hline S. enterica subsp. enterica Enteritidis & RIMD1933006 & + & + \\
\hline S. enterica subsp. enterica Typhimurium & IID1000 & + & + \\
\hline S. enterica subsp. enterica Typhimurium & NBRC12529 & + & + \\
\hline S. enterica subsp. enterica Infantis & ATCC51741 & + & + \\
\hline S. enterica subsp. arizonae & ATCC $13314^{\mathrm{T}}$ & + & + \\
\hline S. bongori & CIP82.33 & + & - \\
\hline \multicolumn{4}{|l|}{$<$ Enterobacteriaceae $>$} \\
\hline Citrobacter freundii & $\mathrm{JCM} 1657^{\mathrm{T}}$ & + & - \\
\hline Citrobacter koseri & JCM1659 & + & - \\
\hline Cronobacter muytjensii & ATCC51329 ${ }^{\mathrm{T}}$ & + & - \\
\hline Enterobacter aerogenes & NCTC $10006^{\mathrm{T}}$ & + & - \\
\hline Ent. cloacae subsp. cloacae & ATCC $13047^{\mathrm{T}}$ & + & - \\
\hline Escherichia coli & $\mathrm{JCM} 1649^{\mathrm{T}}$ & + & - \\
\hline E. coli & NBRC3301 & + & - \\
\hline E. coli & NBRC15034 & + & - \\
\hline E. coli & ATCC51813 & + & - \\
\hline E. coli & ATCC51446 & + & - \\
\hline E. coli $\mathrm{O} 157$ & RIMD0909861 & + & - \\
\hline E. coli $\mathrm{O} 157: \mathrm{H} 7$ & RIMD0909939 & + & - \\
\hline Hafnia alvei & ATCC51815 & + & - \\
\hline Klebsiella pneumoniae subsp. pneumoniae & $\mathrm{ATCC} 13883^{\mathrm{T}}$ & + & - \\
\hline Morganella morganii subsp. morganii & NBRC $3848^{\mathrm{T}}$ & + & - \\
\hline Shigella flexneri & LMG $10473^{\mathrm{T}}$ & + & - \\
\hline S. sonnei & LMG10472 & + & - \\
\hline Yersinia enterocolitica subsp. enterocolitica & ATCC9610 & + & - \\
\hline \multicolumn{4}{|l|}{$<$ Gram negative strains $>$} \\
\hline Aeromonas hydrophila subsp. hydrophila & NBRC3820 & + & - \\
\hline Pseudomonas aeruginosa & ATCC27853 & + & - \\
\hline P. fluorescens & JCM5963 & + & - \\
\hline P. putida & IAM $1236^{\mathrm{T}}$ & + & - \\
\hline Vibrio parahaemolyticus & NBRC $12711^{\mathrm{T}}$ & + & - \\
\hline
\end{tabular}

${ }^{*}$; Type strain, +; positive, -; negative

were observed using epi-fluorescence microscopy (Nikon E600 [Nikon], objective lens; Plan fluor $\times 4$, fluorescent filter; XF204 [Omega] for Alexa 546 ${ }^{\circledR}$ and XF202 [Omega] for Alexa $488^{\circledR}$ ) and images were captured using a digital camera D50 (Nikon). Micro-colony diameter was determined from digital images using ImageJ ver. 1.37 software (National Institute of Health, Bethesda, MD, USA).

Optimization of probe concentration in FISHFC Optimal probe concentration was determined to obtain adequate fluorescence intensity for epi-fluorescence microscopic observation. An aliquot $(0.1 \mathrm{~mL})$ of $S$. Enteritidis NBRC3313 culture was inoculated into $4 \mathrm{~mL}$ of MLCB-broth and then FISHFC was performed as described above. The signal-tonoise $(\mathrm{S} / \mathrm{N})$ ratios were calculated as described previously (Fuchizawa et al., 2008; Taguchi et al., 2006): $\mathrm{S} / \mathrm{N}$ ratio = fluorescence intensity of each micro-colony / fluorescence intensity of the background noise. The fluorescence intensity was measured as a gray-scale value using ImageJ ver. 1.37 software.

Comparison of FISHFC count and conventional plate count in vitro To evaluate FISHFC method accuracy, viable bacterial counts were enumerated using the FISHFC method and the plate count method. $S$. Enteritidis NBRC3313 and 2-fold bacterial count of Citrobacter freundii mixed culture, or $S$. Typhimurium and 8-fold bacterial count of E. coli mixed culture were inoculated into $4 \mathrm{~mL}$ of MLCB broth, and then FISHFC was performed as described above. The probe-associated fluorescent micro-colonies were enumerated using epi-fluorescence microscopy. For the conventional plate count method, Salmonella was enumerated by surface 
plating of $0.1 \mathrm{~mL}$ diluted culture onto MLCB agar (Oxoid), Xylose Lysine Deoxycholate (XLD, Oxoid) agar and Chromagar Salmonella ${ }^{\circledR}$ (CAS, Oxoid). Plates were incubated aerobically at $37^{\circ} \mathrm{C}$ for $24 \mathrm{~h}$ and typical Salmonella colonies were enumerated. When typical Salmonella colonies were present on the selective agar after $24 \mathrm{~h}$ of incubation, they were picked and inoculated on triple sugar iron (TSI) agar and lysine indole production motility (LIM) agar (Nissui Pharmaceutical Co.Ltd. Tokyo, Japan) for confirmation.

Enumeration of Salmonella in vegetable samples using FISHFC method Food samples (radish sprouts, potherb mustard, tomato, spinach, celery, cut cabbage and potato salad) were purchased from a local supermarket. Individual vegetable and processed food samples $(25 \mathrm{~g})$ were artificially spiked with various volumes $(0.1-1.0 \mathrm{~mL})$ of Salmonella mixed culture $\left(10^{4}-10^{5} \mathrm{CFU} / \mathrm{mL}\right.$ containing $S$. Enteritidis NBRC3313, $S$. Typhimurium IID1000 and $S$. Infantis ATCC51741). Spiked food samples were then homogenized with $225 \mathrm{~mL}$ of buffered peptone water for $30 \mathrm{~s}$ using a Stomacher 400 circulator (Seward Medical, London, UK). For FISHFC, $0.1 \mathrm{~mL}$ of food homogenate was mixed with $4 \mathrm{~mL}$ of MLCB broth and then filtered using 37-mm monitor units (Advantec) fitted with hydrophilic polypropylene membranes, and FISHFC was performed as described above. Probe-associated fluorescent micro-colonies were counted using epi-fluorescence microscopy. For determination using conventional plate count methods, Salmonella was enumerated by surface plating of diluted food homogenate onto MLCB, XLD and CAS agars. Plates were incubated aerobically at $37^{\circ} \mathrm{C}$ for $24 \mathrm{~h}$ and typical Salmonella colonies were confirmed with TSI and LIM agars and enumerated. All determinations were performed two to six times each.

\section{Results}

Probe design and specificity for Salmonella detection

SAL343 was designed for $S$. enterica detection from multiple alignments of 23S rRNA sequences of Salmonella and other related strains (Table 2). Homologous regions were searched using the web server probeCheck (Loy et al., 2008), confirming that the species-specificity of SAL343 completely matched only S. enterica. However, SAL343 also had four mismatch base pairs compared to closely related bacterial sequences (E. coli, Shigella dysenteriae and others, Table 2). FISHFC specificity using SAL343 was assessed with the micro-colonies of the various bacteria listed in Table 1 . The specificity test results revealed that SAL343 was highly specific for $S$. enterica, and did not react with other serotypes of Salmonella, including S. bongori (Table 1). All microcolonies of tested strains were hybridized with EUB338 and the fluorescence was observed as a positive control.

Optimization of FISHFC protocol for Salmonella detection To determine a suitable cultivation time for the FISHFC method, the micro-colony size of three $S$. enterica strains on the filters was investigated (Fig. 1). For cultures inoculated with Salmonella cells, micro-colonies showing probeassociated fluorescence were visible (at a magnification of 40-fold) after $6 \mathrm{~h}$ incubation. Average micro-colony diameter for each culture was determined at 6,7 and $8 \mathrm{~h}$ incubation. The micro-colony diameter at 6,7 and $8 \mathrm{~h}$ incubation was 47.6, 108.6 and $246.8 \mu \mathrm{m}$ for $S$. Enteritidis NBRC3313, 62.4, 82.7 and $157.7 \mu \mathrm{m}$ for $S$. Enteritidis RIMD1933001, 42.3, 78.3 and $152.3 \mu \mathrm{m}$ for $S$. Typhimurium IID1000, and 65.1, 106.2 and $156.3 \mu \mathrm{m}$ for $S$. Typhimurium NBRC12528, respectively. The micro-colony diameters after $8 \mathrm{~h}$ incubation were easily distinguishable from those of 6 and $7 \mathrm{~h}$ incubation. On the other hand, food particles microscopically

Table 2. Sequence alignments of Salmonella and related species with the oligonucleotide sequence of SAL343.

\begin{tabular}{|c|c|c|c|c|c|c|c|c|c|c|c|c|c|c|c|c|c|c|c|c|c|c|c|}
\hline & \multicolumn{23}{|c|}{ SAL343 probe sequence } \\
\hline & & $\begin{array}{r}3^{\prime}-\mathrm{G} \\
343\end{array}$ & $\mathrm{~T}$ & $\mathrm{~T}$ & $\mathrm{~T}$ & $\mathrm{~T}$ & C & G & $\mathrm{C}$ & G & $\mathrm{T}$ & A & $\mathrm{C}$ & A & $\mathrm{C}$ & G & A & $\mathrm{C}$ & A & $\mathrm{C}$ & & & $\begin{array}{l}\text { G }-5 \text { ' } \\
364\end{array}$ \\
\hline & (Acc. No.) & $\downarrow$ & & & & & & & & & & & & & & & & & & & & & $\downarrow$ \\
\hline Salmonella Enteritidis & EU146952 & $\mathrm{C}$ & A & A & A & A & G & $\mathrm{C}$ & G & $\mathrm{C}$ & A & $\mathrm{U}$ & G & $\mathrm{U}$ & G & $\mathrm{C}$ & $\mathrm{U}$ & G & $\mathrm{U}$ & G & A & G & $\mathrm{C}$ \\
\hline Escherichia coli & EU146962 & . & . & . & . & . & $\mathrm{A}$ & $\mathrm{U}$ & & . & . & $\mathrm{C}$ & A & . & . & . & . & . & . & . & . & . & . \\
\hline Escherichia coli $\mathrm{O} 157: \mathrm{H} 7$ & AB035924 & . & . & . & . & . & A & $\mathrm{U}$ & . & . & . & $\mathrm{C}$ & A & . & . & . & . & . & . & . & . & . & . \\
\hline Escherichia albertii & ABKX01000024 & . & . & . & . & . & A & $\mathrm{U}$ & . & . & . & $\mathrm{C}$ & A & . & . & . & . & . & . & . & . & . & . \\
\hline Shigella dysenteriae & AAMJ01000007 & . & . & . & . & . & A & $\mathrm{U}$ & . & . & . & $\mathrm{C}$ & A & . & . & . & . & . & . & . & . & . & . \\
\hline Erwinia tasmaniensis & CU468135 & . & . & . & . & . & A & $\mathrm{U}$ & . & . & . & $\mathrm{C}$ & $\mathrm{U}$ & . & . & . & . & . & . & . & . & . & $\mathrm{U}$ \\
\hline Yersinia entrocolitica & U77925 & . & . & . & . & . & A & $\mathrm{U}$ & . & . & . & . & A & . & . & $\mathrm{U}$ & . & . & . & . & . & . & $\mathrm{U}$ \\
\hline Salmonella bongori & U77927 & . & G & . & . & . & A & $\mathrm{U}$ & . & . & . & $\mathrm{C}$ & $\mathrm{C}$ & . & . & . & . & . & . & . & . & . & . \\
\hline Citrobacter freundii & U77928 & . & . & . & . & . & A & $\mathrm{U}$ & . & . & . & $\mathrm{C}$ & A & G & . & $\mathrm{U}$ & . & . & . & . & . & A & . \\
\hline
\end{tabular}

*Identical residues with Salmonella Enteritidis are indicated as dots. 


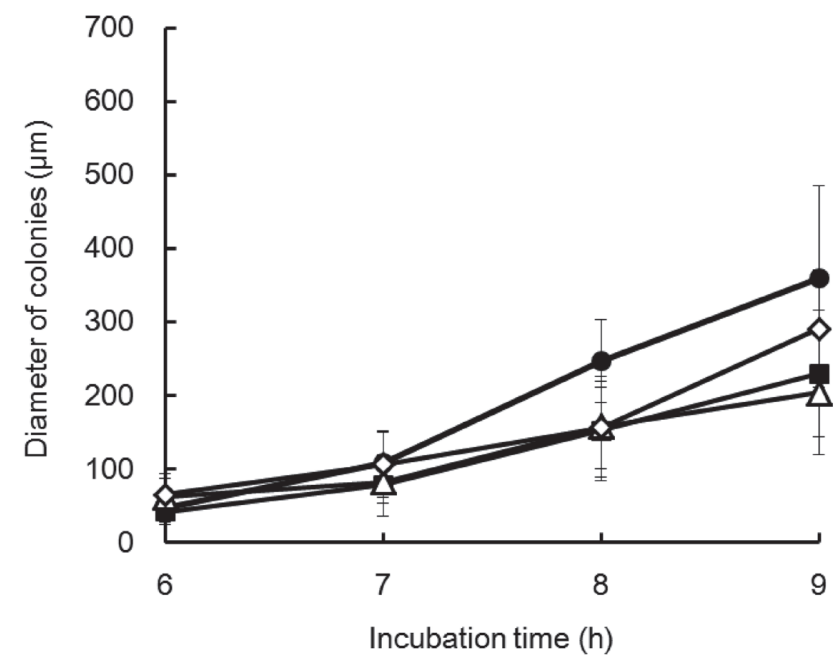

Fig. 1. Colony size of $S$. enterica strains incubated for different periods.

Salmonella micro-colonies were hybridized after incubation in MLCB medium. Colony size on FISHFC images was analyzed with ImageJ. Symbols: @, S. Enteritidis NBRC3313; $\mathbf{\square}, S$. Enteritidis RIMD1933001; $\triangle, S$. Typhimurium NBRC12528; $\diamond, S$. Typhimurium IID1000.

observed using 40-fold magnification were $59.6 \pm 32.5 \mu \mathrm{m}$ in average diameter (Fig. 2). These findings suggest that a $>$ $100-\mu \mathrm{m}$ micro-colony diameter is required in FISHFC detection of Salmonella in order to distinguish micro-colonies from food particles. Therefore, the optimal incubation time for Salmonella spp. was determined to be $37^{\circ} \mathrm{C}$ for $8 \mathrm{~h}$.

In addition, the optimal probe concentration in FISHFC was investigated (Fig. 3). After FISHFC detection with various probe concentrations $(31.3-1000 \mathrm{nM})$, high fluorescence $\mathrm{S} / \mathrm{N}$ ratios $(>4.0)$ were observed at low probe

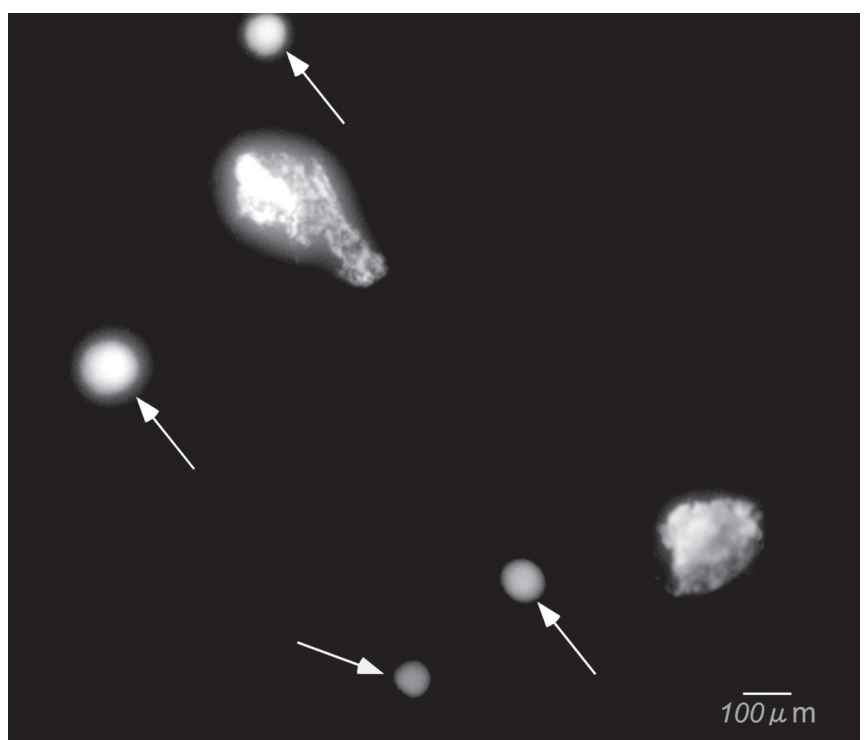

Fig. 2. Fluorescent image of Salmonella micro-colonies and food particles using FISHFC detection.

The arrows indicate $S$. enterica micro-colonies.

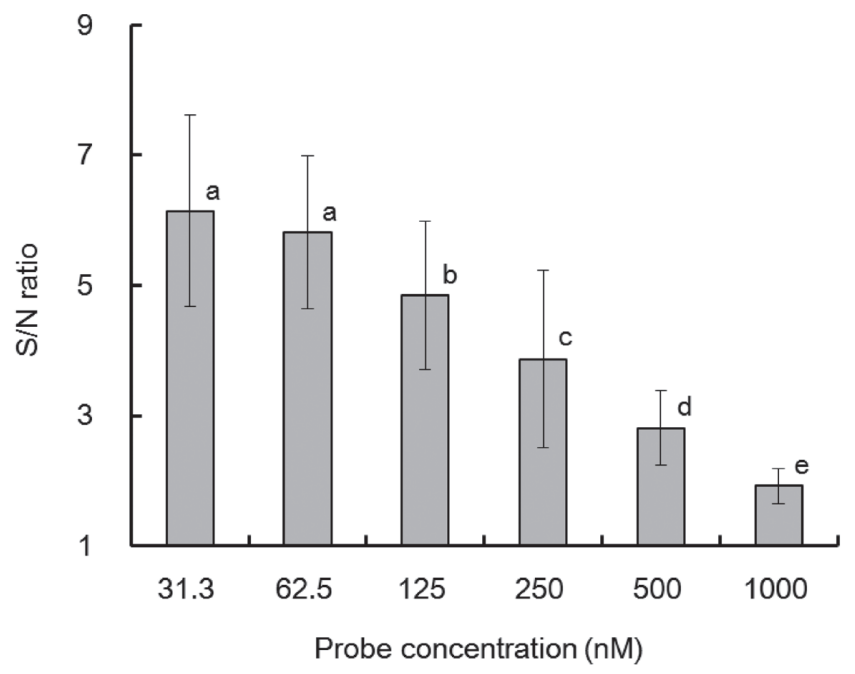

Fig. 3. Influence of probe concentration on the $\mathrm{S} / \mathrm{N}$ ratios of probe-associated fluorescent colonies using FISHFC detection.

Bars marked with different letters indicate statistically significant differences $(p>0.05)$.

concentrations $(31.3-125 \mathrm{nM})$. Specifically, low probe concentrations (31.3 and $62.5 \mathrm{nM}$ ) resulted in higher $\mathrm{S} / \mathrm{N}$ ratios $(>6.0)(\mathrm{S} / \mathrm{N}$ ratio $=6.2$ and 6.0 , respectively). The $\mathrm{S} / \mathrm{N}$ ratios with low probe concentrations (31.3 and $62.5 \mathrm{nM})$ were not significantly different $(p>0.05)$. Thus, the optimal probe concentration in FISHFC was determined to be $62.5 \mathrm{nM}$.

Quantitative validation between FISHFC count and conventional plate counts Viable $S$. enterica count determined by FISHFC was compared to that obtained by conventional plate count methods using selective agar (MLCB, XLD and CAS). In this study, food samples artificially contaminated with Salmonella, as well as other related bacteria, were used. Artificial contamination was conducted to assess the influence of naturally occurring microorganisms on the accuracy of bacterial detection. When inoculating $S$. Enteritidis, twofold of $C$. freundii culture was also inoculated and incubated at $37^{\circ} \mathrm{C}$ for $6-8 \mathrm{~h}$. After $8 \mathrm{~h}$ of incubation, the FISHFC method detected $2.6 \pm 0.1 \log \mathrm{CFU} / \mathrm{mL}$ and convention plate counts with MLCB agar detected $2.7 \pm 0.1 \log \mathrm{CFU} / \mathrm{mL}$. No significant differences between the FISHFC and MLCB agar methods were observed (Table $3, p>0.05$ ). On the other

Table 3. Comparison of FISHFC counts using SAL343 and plate count methods.

\begin{tabular}{ccccc}
\hline & \multicolumn{4}{c}{ Salmonella count $(\log$ CFU / mL) } \\
\cline { 2 - 5 } & SAL343 & MLCB & XLD & CAS \\
\hline Sample A & $2.6 \pm 0.1$ & $2.7 \pm 0.1$ & $1.1 \pm 0.3$ & $1.5 \pm 0.3$ \\
Sample B & $2.3 \pm 0.1$ & $2.5 \pm 0.2$ & $2.1 \pm 0.2$ & $2.5 \pm 0.2$ \\
\hline
\end{tabular}

Sample A spiked $S$. Enteritidis : $C$. freundii $=1: 2$

Sample Bspiked $S$. Typhimurium : E. coli $=1: 8$ 


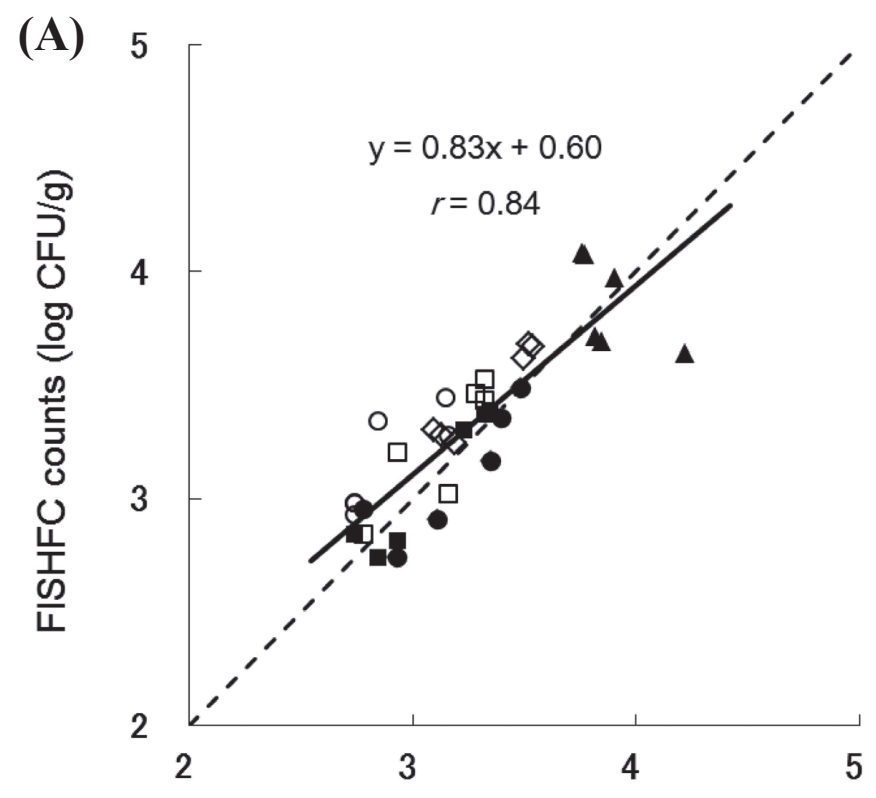

Plate counts (log CFU/g)

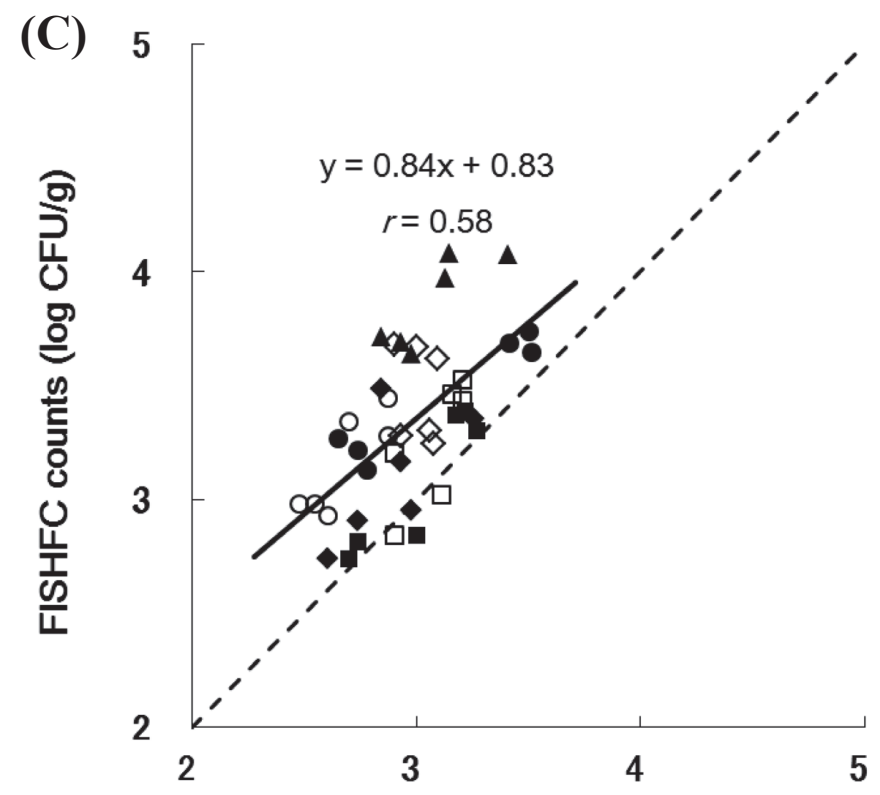

Plate counts (log CFU/g)

hand, the conventional plate count methods with XLD and CAS agars detected $1.1 \pm 0.3$ and $1.5 \pm 0.3 \log \mathrm{CFU} / \mathrm{mL}$, respectively. When $S$. Typhimurium was artificially inoculated, 8 -fold of $E$. coli culture was also inoculated and incubated at $37^{\circ} \mathrm{C}$ for $6-8 \mathrm{~h}$. After $8 \mathrm{~h}$ of incubation, the FISHFC method detected $2.3 \pm 0.1 \log \mathrm{CFU} / \mathrm{mL}$ and conventional plate counts with CAS, MLCB and XLD agars detected $2.5 \pm$ $0.2,2.5 \pm 0.2$ and $2.1 \pm 0.2 \log \mathrm{CFU} / \mathrm{mL}$, respectively. These results indicate that FISHFC is equally able to detect viable Salmonella in artificially contaminated samples as conventional agar plating methods.

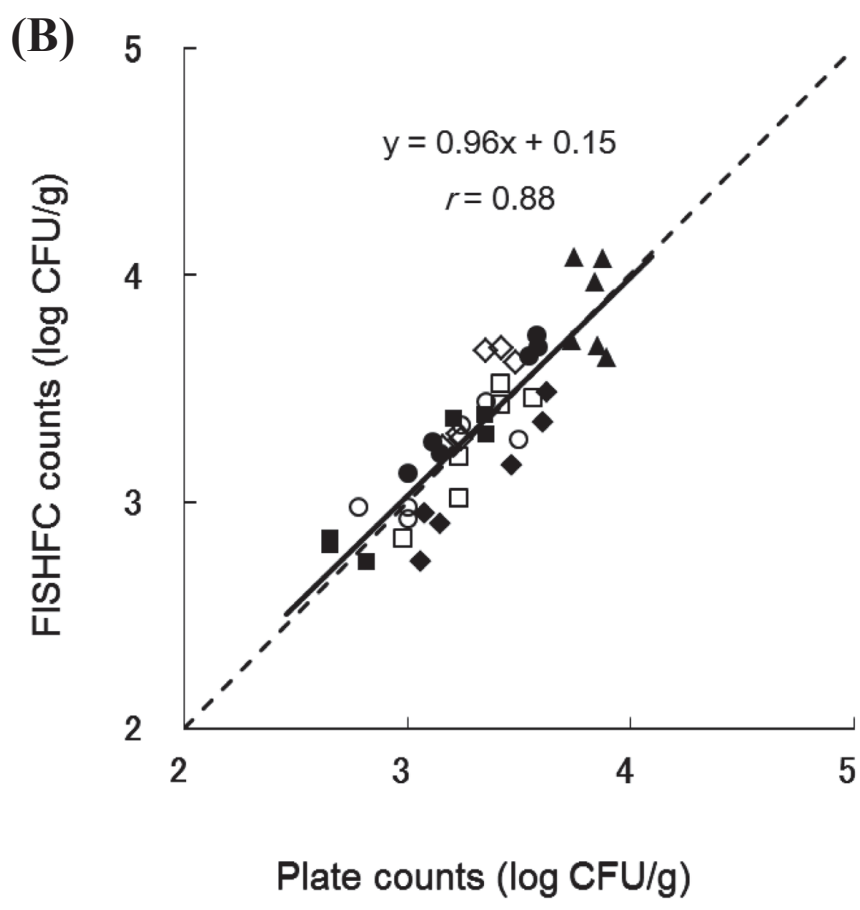

Fig. 4. Correlation between Salmonella viable counts in artificially inoculated food detected using the FISHFC method and three conventional plate count methods.

The linear correlation equation of MLCB agar (Fig. 4A) was y = $0.83 \mathrm{x}+0.60(r=0.84)$. The linear correlation equation of XLD agar (Fig. 4B) was $\mathrm{y}=0.96 \mathrm{x}+0.15(r=0.88)$. The linear correlation equation of CAS agar (Fig. 4C) was $\mathrm{y}=0.84 \mathrm{x}+0.83(r=$ 0.58 ). Food samples are $\bigcirc$, tomato; $\bigcirc$, potato salad; $\square$, spinach; $\mathbf{\square}$, potherb mustard; $\boldsymbol{\Delta}$, radish sprouts; $\diamond$, celery; $\diamond$, cut cabbage (all samples $n=6$ ).

Comparison of FISHFC method and CCBM for Salmonella enumeration in fresh vegetable samples Viable Salmonella counts in artificially inoculated fresh food samples were determined using FISHFC and conventional plate count methods with Salmonella selective agar (Fig. 4). The initial total viable bacterial counts in the tested vegetables were recorded as $2.7-6.5 \log \mathrm{CFU} / \mathrm{g}$. For different vegetable samples, paired t-tests revealed no significant differences between FISHFC count and conventional plate counts with MLCB and XLD agars $(p>0.05)$, while FISHFC count was significantly higher than plate count with CAS $(p<0.05)$ agar. In this experiment, the linear correlation equation and the correlation coefficient were $\mathrm{y}=0.83 \mathrm{x}+0.60$ and $r=0.84$ between FISHFC count and plate count with MLCB (Fig. $4 \mathrm{~A}), \mathrm{y}=0.96 \mathrm{x}+0.15$ and $r=0.88$ with XLD (Fig. 4B), $\mathrm{y}=$ $0.84 \mathrm{x}+0.83$ and $r=0.58$ with CAS (Fig. 4 C) agars, respectively.

\section{Discussion}

In this study, a region that is specific for $S$. enterica was found between positions 343 and 364 (E. coli rRNA num- 
bering system). A region similar to that targeted by SAL343 was previously used in PCR primer and FISH probe development for Salmonella detection (Fang et al., 2003; Rönner and Stackebrandt, 1994). Amann et al. (1995) reported that 23S rRNA was often used as a hybridization target molecule, because its sequences showed higher phylogenetic specificity than 16S rRNA sequences.

Yilmaz and Noguera (2004) reported that fluorescence intensity was negatively correlated to the Gibbs free energy change $(\Delta G)$ in hybridization between the DNA probe and rRNA. Sawabe et al. (2009) showed that greater brightness from the hybridized probes was obtained when $V$. parahaemolyticus was hybridized with probes in which $\Delta G$ ranged from -17 to -22 ; therefore, SAL343 was designed accordingly. The $\Delta G$ value of SAL343 was calculated at $-25.41 \mathrm{kcal} / \mathrm{mol}$, as compared that of probe Sal-1 (Fang et al., 2003), which was $-22.45 \mathrm{kcal} / \mathrm{mol}$. A homology search was conducted using the web server probeCheck (Loy et al., 2008) and SAL343 species-specificity was confirmed for the target species, as described in the Results. Probe specificity results showed species-specific and efficient determination of S. enterica by the FISH technique. On the other hand, highly selective MLCB, XLD agars or chromogenic media, such as CAS, have been using to detect or isolate Salmonella from various samples. MLCB is used principally for Salmonella detection in food and environmental samples, due to its high selectivity (Nye et al., 2002; van Schothorst et al., 1987). On the other hands, XLD is the most commonly used medium for isolating S. enterica (Nye et al., 2002), and CAS is superior to other selective agars for isolating Salmonella from stool samples (Gailot et al., 1999; Maddocks et al., 2002; Perez et al., 2003).

In this study, the Salmonella viable count obtained from MLCB was the highest among the three selective agars (MLCB, XLD and CAS) employed, and was not significant different from the FISHFC count. The cultivation time for micro-colonies on the membrane filters is a crucial point in the FISHFC method. With FISHFC Salmonella detection, the probe-associated fluorescence of micro-colonies appeared after $6 \mathrm{~h}$ incubation under epi-fluorescence microscopic observation using a 40-fold magnification; however, these micro-colonies were small and difficult to distinguish from the auto-fluorescence of food particles. Notably, micro-colony size increased to $>100 \mu \mathrm{m}$ after $8 \mathrm{~h}$ incubation and their probe-associated fluorescence was easily distinguishable from that of food particles, due to differences in fluorescence intensity and the shape of fluorescent signals (Fig. 2). A previous report showed that a micro-colony diameter size $>100$ $\mu \mathrm{m}$ was necessary to detect $C$. perfringens using FISHFC (Shimizu et al., 2009). Also, in this FISHFC Salmonella de- tection method, a bright fluorescence signal associated with the probe could be obtained without permeabilization of the micro-colonies after ethanol fixation, as described in previous reports (Fuchizawa et al., 2008, 2009; Shimizu et al., 2009). Generally, the hybridization signal intensity may be affected by the bacterial cell ribosome content (Amann et al., 1995), and the amount of rRNA in the cell is dependent on incubation time (Kerkhof and Kemp, 1999). Also, in some cases, permeabilization of the bacterial cell membrane by enzyme treatment may be necessary for probe access into the cells. However, with respect to FISHFC technology, bacterial micro-colonies hybridized with fluorescent probes generate stronger fluorescence signals as a result of the aggregation of fluorescent single cells. Therefore, fluorescent microcolonies associated with the probe are easily detected with microscopic observation in the FISHFC method, even at low magnification.

Optimal probe concentration in Salmonella detection was investigated, and the fluorescence intensity of the samples with FISHFC detection using various SAL343 concentrations was compared. High $\mathrm{S} / \mathrm{N}$ ratios $(>6.0)$ for microscopic observation were obtained when low concentrations of SAL343 were used with FISH, especially at $31.3 \mathrm{nM}$ and $62.5 \mathrm{nM}$. However, the fluorescence signal at $31.3 \mathrm{nM}$ was darker than that at $62.5 \mathrm{nM}$ (data not shown). On the other hand, high probe concentrations resulted in a low $\mathrm{S} / \mathrm{N}$ ratio, due to high background fluorescence. In previous reports, the FISHFC method was performed using a final probe concentration of $1-10 \mu \mathrm{M}$ in hybridization buffers (Fuchizawa et al., 2008, 2009; Ootsubo et al., 2003; Sawabe et al., 2009; Shimizu et al., 2009). A S/N ratio of at least 1.3 was required for automatic enumeration of probe-stained planktonic cells on the filter (Pernthaler et al., 2003), while a $\mathrm{S} / \mathrm{N}$ ratio of 3.0 was necessary to detect probe-stained $E$. coli single cells in activated sludge (Hoshino et al., 2008). Therefore, in this study, an optimal probe concentration of $62.5 \mathrm{nM}$ was employed to detect Salmonella micro-colonies without affecting specificity.

To date, many rapid detection methods based on molecular biology have been developed; however, many of these have limitations. Micro-colony staining methods using fluorescent dyes, such as DAPI, have been developed for counting total viable bacteria in the environment (Kepner and Pratt, 1994; Rodrigues and Kroll, 1988; Wang et al., 2007). These methods are rapid and convenient, but cannot directly identify the detected microorganisms. Single-cell FISH methods have proven to be a valuable tool in the study and identification of specific microorganisms in environmental and clinical samples (Bottari et al., 2006). However, the practical application of these methods for food and environmental samples is lim- 
ited, due to the interference of food particles with bacterial cell counts under microscopic observation. While ELISA has an advantage over plate count methods, in that it can deal with multiple samples, it is not quantitative and cannot detect low bacterial contamination levels (McCourt et al., 2005). In spite of its specificity and rapidity, disadvantages of PCRbased methods include the requirement of a DNA extraction step, the detection of both live and dead cells (Gurjar et al., 2008; Wise and Siragusa, 2005), and reaction interference by certain chemical inhibitors, such as polyphenolic compounds, in complex substrates (Koonjul et al., 1999; Wise and Siragusa, 2005). To overcome these limitations, Ootsubo et al. (2003) developed a FISHFC method and showed specific detection and quantification of cultivable Enterobacteriaceae from food and environmental water. The FISHFC method achieved results within $7 \mathrm{~h}$, and enteric pathogens in ground meat could be detected and enumerated at contamination levels $>2 \log$ CFU/g. Shimizu et al. (2009) showed that FISHFC was an equally accurate yet faster method than the conventional plate count method when enumerating viable $C$. perfringens in ground beef. Fuchizawa et al. (2009) also showed that FISHFC could be used to enumerate viable L. monocytogenes in smoked salmon and Camembert cheese.

In conclusion, the FISHFC method using SAL343, developed in this study, exhibits equivalent detection accuracy to the conventional plate count method. Furthermore, this method enumerated only viable (colony-formative) $S$. enterica from food samples within $10 \mathrm{~h}$, suggesting that the evaluation of $S$. enterica contamination can be completed within a single working day. In comparison, enumeration of Salmonella by conventional plate count with selective agars (MLCB, XLD and CAS) is time-consuming and labor-intensive, and may be unsuitable for the rapid estimation required in food hygiene and sanitation. Salmonella detection using the FISHFC method with SAL343 could contribute to ensuring food safety, especially of fresh vegetable products.

Acknowledgements This work was supported by a research project ensuring food safety from farm to table (DI-7302), which was funded by the Ministry of Agriculture, Forestry and Fisheries (MAFF) of Japan.

\section{References}

Agbaja, M., Begum, R.H., Oyekunle, M.A., Ojo, O.E. and Adenubi, O.T. (2011). Evolution of Salmonella nomenclature: a critical note. Folia Microbiol., 56, 497-503.

Amann, R.I., Krumholz, L. and Stahl, D.A. (1990). Fluorescentoligonucleotide probing of whole cells for determinative, phylogenetic, and environmental studies in microbiology. J. Bacteriol., 172, 762-770.
Amann, R.I., Ludwig, W. and Schleifer, K.H., (1995). Phylogenetic identification and in situ detection of individual microbial cells without cultivation. Microbiol. Rev., 59, 143-169.

Bottari, B., Ercolini, D., Gatti, M. and Neviani, E. (2006). Application of FISH technology for microbiological analysis: current state and prospects. Appl. Microbiol. Biotechnol., 73, 485-494.

Fang, Q., Brockmann, S., Botzenhart, K. and Wiedenmann, A. (2003). Improved detection of Salmonella spp. in foods by fluorescent in situ hybridization with 23S rRNA probes: a comparison with conventional culture methods. J. Food Prot., 66, 723-731.

Fuchizawa, I., Shimizu, S., Kawai, Y. and Yamazaki, K. (2008). Specific detection and quantitative enumeration of Listeria spp. using fluorescent in situ hybridization in combination with filter cultivation (FISHFC). J. Appl. Microbiol., 105, 502-509.

Fuchizawa, I., Shimizu, S., Ootsubo, M., Kawai, Y. and Yamazaki, K. (2009). Specific and rapid quantification of viable Listeria monocytogenes using fluorescence in situ hybridization in combination with filter cultivation. Microbes Environ., 24, 273-275.

Giammanco, G.M., Pignato, S., Mammina, C., Grimont, F., Grimont, P.A., Nastasi, A. and Giammanco, G. (2002). Persistent endemicity of Salmonella bongori 48:z(35): -- in Southern Italy: molecular characterization of human, animal, and environmental isolates. J. Clin. Microbiol., 40, 3502-3505.

Goodridge, C., Goodridge, L., Gottfried, D., Edmonds, P. and Wyvill, J.C. (2003). A rapid most-probable-number-based enzyme-linked immunosorbent assay for the detection and enumeration of Salmonella Typhimurium in poultry wastewater. $J$. Food Prot., 66, 2302-2306.

Gurjar, A.A., Hegde, N.V., Love, B.C. and Jayarao, B.M. (2008) Real-time multiplex PCR assay for rapid detection and toxintyping of Clostridium perfringens toxin producing strains in feces of dairy cattle. Mol. Cell. Probes, 22, 90-95.

Hoshino, T., Yilmaz, L.S., Noguera, D.R., Daims, H. and Wagner, M. (2008). Quantification of target molecules needed to detect microorganisms by fluorescence in situ hybridization (FISH) and catalyzed reporter deposition-FISH. Appl. Environ. Microbiol., 74, 5068-5077.

Kawasaki, S., Fratamico, P.M., Horikoshi, N., Okada, Y., Takeshita, K., Sameshima, T. and Kawamoto, S. (2010). Multiplex real-time polymerase chain reaction assay for simultaneous detection and quantification of Salmonella species, Listeria monocytogenes, and Escherichia coli $\mathrm{O} 157: \mathrm{H} 7$ in ground pork samples. Foodborne Pathog. Dis., 7, 549-554.

Kepner, R.L. and Pratt, J.R. (1994). Use of fluorochromes for direct enumeration of total bacteria in environmental samples: past and present. Microbiol. Rev., 58, 603-615.

Kerkhof, L. and Kemp, P. (1999). Small ribosomal RNA content in marine Proteobacteria during non-steady-state growth. FEMS Microbiol. Ecol., 30, 253-260.

Kim, J.S., Lee, G.G., Park, J.S., Jung, Y.H., Kwak, H.S., Kim, S.B., 
Nam, Y.S. and Kwon, S.T. (2007). A novel multiplex PCR assay for rapid and simultaneous detection of five pathogenic bacteria: Escherichia coli 0157:H7, Salmonella, Staphylococcus aureus, Listeria monocytogenes, and Vibrio parahaemolyticus. J. Food Prot., 70, 1656-1662.

Koonjul, P.K., Brandt, W.F., Farrant, J.M. and Lindsey, G.G. (1999). Inclusion of polyvinylpyrrolidone in the polymerase chain reaction reverses the inhibitory effects of polyphenolic contamination of RNA. Nucleic Acids Res., 27, 915-916.

Loy, A., Arnold, R., Tischler, P., Rattei, T., Wagner, M. and Horn, M. (2008). probeCheck -- a central resource for evaluating oligonucleotide probe coverage and specificity. Environ. Microbiol., 10, 2894-2898.

Maddocks, S., Olma, T. and Chen, S. (2002). Comparison of CHROMagar Salmonella medium and xylose-lysine-desoxycholate and Salmonella-Shigella agars for isolation of Salmonella strains from stool samples. J. Clin. Microbiol., 40, 2999-3003.

Matthews, K.R. (2006). Microorganisms associated with fruits and vegetables. In "Microbiology of fresh produce," ed. by K. R. Matthews. ASM Press, Washington, DC, pp.1-9.

McCourt, M.T., Finlay, D.A., Laird, C., Smyth, J.A., Bell, C. and Ball, H.J. (2005). Sandwich ELISA detection of Clostridium perfringens cells and alpha-toxin from field cases of necrotic enteritis of poultry. Vet. Microbiol., 106, 259-264.

Nye, K.J., Fallon, D., Frodsham, D., Gee, B., Graham, C., Howe, S., Messer, S., Turner, T. and Warren, R.E. (2002). An evaluation of the performance of XLD, DCA, MLCB, and ABC agars as direct plating media for the isolation of Salmonella enterica from faeces. J. Clin. Pathol., 55, 286-288.

Ootsubo, M., Shimizu, T., Tanaka, R., Sawabe, T., Tajima, K. and Ezura, Y. (2003). Seven-hour fluorescence in situ hybridization technique for enumeration of Enterobacteriaceae in food and environmental water sample. J. Appl. Microbiol., 95, 1182-1190.

Pernthaler, J., Pernthaler, A. and Amann, R. (2003). Automated enumeration of groups of marine picoplankton after fluorescence in situ hybridization. Appl. Environ. Microbiol., 69, 2631-2637.

Perez, J.M., Cavalli, P., Roure, C., Renac, R., Gille, Y. and Freydiere, A.M. (2003). Comparison of four chromogenic media and Hektoen agar for detection and presumptive identification of
Salmonella strains in human stools. J. Clin. Microbiol., 41, 11301134.

Rodrigues, U.M. and Kroll, R.G. (1988). Rapid selective enumeration of bacteria in foods using a microcolony epifluorescence microscopy technique. J. Appl. Bacteriol., 64, 65-78.

Rönner, G.E. and Stackebrandt, E. (1994). Development of 23S rDNA-oligonucleotide probes for the identification of Salmonella species. Syst. Appl. Microbiol., 17, 257-264.

Sawabe, T., Yoshizawa, A., Kawanishi, Y., Komatsu-Takeda, E., Nakagawa, S., Sawabe, T., Ootubo, M., Satomi, M., Yano, Y. and Yamazaki, K. (2009). Multi-probe-fluorescence in situ hybridization for the rapid enumeration of viable Vibrio parahaemolyticus. Microbes Environ., 24, 259-264.

Shimizu, S., Ootsubo, M., Kubosawa, Y., Fuchizawa, I., Kawai, Y. and Yamazaki, K. (2009). Fluorescent in situ hybridization in combination with filter cultivation (FISHFC) method for specific detection and enumeration of viable Clostridium perfringens. Food Microbiol., 26, 425-431.

Su, L.H. and Chiu, C.H. (2007). Salmonella: Clinical importance and evolution of nomenclature. Chang Gung Med J., 30, 210219.

Taguchi, T., Shinozaki, Y., Takeyama, H., Haraguchi, S., Yoshino, M., Kaneko, M., Ishimori, Y. and Matsunaga, T. (2006). Direct counting of Cryptosporidium parvum oocysts using fluorescence in situ hybridization on a membrane filter. J. Microbiol. Methods, 67, 373-380.

van Schothorst, M., Renaud, A. and van Beek, C. (1987). Salmonella isolation using RVS broth and MLCB agar. Food Microbiol., 4, 11-18.

Wang, X., Yamaguchi, N., Someya, T. and Nasu, M. (2007). Rapid and automated enumeration of viable bacteria in compost using a micro-colony auto counting system. J. Microbiol. Methods, 71, 1-6.

Wise, M.G. and Siragusa, G.R. (2005). Quantitative detection of Clostridium perfringens in the broiler fowl gastrointestinal tract by real-time PCR. Appl. Environ. Microbiol., 71, 3911-3916.

Yilmaz, L.S. and Noguera, D.R. (2004). Mechanistic approach to the problem of hybridization efficiency in fluorescent in situ hybridization. Appl. Environ. Microbiol., 70, 7126-7139. 